\title{
The Distribution of Foreign Language Skills as a Game Equilibrium
}

\author{
Reinhard Selten \\ Jonathan Pool
}

Originally published in, and reprinted with corrections with kind permission of Springer Science and Business Media from:

Reinhard Selten (ed.), Game Equilibrium Models IV: Social and Political Interaction (Berlin: Springer-Verlag, 1991), pp. 64-87.

(C) Springer-Verlag Berlin · Heidelberg 1991

\section{Utilika Foundation}

914 NW 57th Street

Seattle, Washington 98107-2847

Telephone (206) 801-1906

http://utilika.org 


\title{
The Distribution of Foreign Language Skills as a Game Equilibrium
}

\author{
Reinhard Selten \\ Jonathan Pool
}

\section{Introduction}

The birth, death, growth, and shrinkage of languages over millennia has given us a world containing about three thousand living languages, whose speakers number from 1 up to several hundred million. Our current knowledge of what causes a language to gain more speakers than it loses or lose more speakers than it gains is limited to a few generalizations about bivariate, more-less effects (see Dressler, 1982; Laponce, 1984; Lieberson, 1982). One important generalization is that the children of two native speakers of the same language tend to acquire that native language unless outside the home the language is rarely used or is despised. In addition, persons who spend a few years or more in a milieu (e.g., neighborhood, school, or workplace) where a language other than their native language is the main language tend to add the other language to their repertoire. Persons tend to learn a language through deliberate study (in contrast with immersion in its milieu) when the language is spoken by many persons, has widely distributed speakers, has wealthy and powerful speakers, and has a prestigious literature, art, and history. Languages tend to lose speakers through death, of course, but also through forgetting by their native and nonnative speakers. Forgetting tends to take place among persons who are not in contact with other speakers of the language or whose rewards for using the language are small or negative. There is little evidence as to whether the difficulty of a language or its effectiveness as an instrument of thought and communication influences its acquisition of new speakers or its loss of former speakers.

As Vaillancourt (1985, p. 18) points out, almost all attempts to model aspects of the distribution of language skills in a population have started with the assumption that this distribution is fixed. Given a population distribution of language skills and some mechanism whereby costs of production or benefits of consumption depend on this distribution, one can derive predictions about the production and consumption of linguistically specialized products (Hočevar, 1975; Vaillancourt, 1985a), the earnings of persons with different repertoires of language skills (Sabourin, 1985; Vaillancourt \& Lacroix, 1985), and the distributions of language skills within labor markets and firms (Breton \& Mieskowski, 1975; Sabourin, 1985). 
In the short term, this assumption of a fixed language-skill distribution in a population is realistic, but over a period of years the distribution of language skills changes, partly as a consequence of choices that persons make about which languages to impart to their children, which languages to learn, and how thoroughly to learn languages. Grenier (1985) and Lang (1986) have attempted to model such choices.

Grenier's (1985) model of a two-language economy assumes that each person's earnings level is a function of (1) the person's native language, (2) whether the person is bilingual, and (3) other attributes of the person. A person is assumed to be bilingual if and only if the present value of the increase in earnings for that person resulting from being bilingual exceeds the present value of the cost to that person of becoming bilingual. Using some additional assumptions about the form of the earnings function, the model estimates what the earnings of any member of a population would be if that member's monolingual/bilingual status were reversed and all the other attributes of the person were held constant. The Grenier model uses population data to estimate the parameters of the prediction equation, and for any set of population data it derives parameters. Thus, the model does not make falsifiable predictions of the distribution of language skills in a population. It does not predict that any distribution of language skills is by itself impossible, and it does not predict that any distribution of language skills is incompatible with some distribution of other attributes.

Lang's (1986) first model of a two-language labor market in equilibrium assumes that all employers have the same native language. Employers choose whether to hire workers with the same native language as theirs or the other native language. In the latter case, either these workers must be bilingual or the employer must become bilingual. The wage of bilingual workers is assumed to compensate them exactly for the cost of becoming bilingual. In a more elaborate model, Lang assumes that most but not all employers have the same native language, and that employers hire not only workers but also supervisors. The workers are assumed to be monolingual, and the supervisor in any firm must be bilingual if the employer, the supervisor, and the workers do not all have the same native language. Supervisors' wages compensate them exactly for their supervisorial training, and bilingual supervisors are further compensated exactly for the cost of learning the second language. Lang finds in both models that only native speakers of the language that has few or no employers become bilingual. Their workers are paid less, while their supervisors are paid more, than native speakers of the language that has most or all of the employers. One peculiarity of Lang's models is their treatment of the linguistic distribution of employers as fixed. The overrepresentation of employers with one of the two native languages, combined with the assumed need for employers and their employees to understand one another, creates linguistically differentiated wage rates. The lower wages paid to workers of one native language makes it possible for the employers having that native language to make higher profits. Lang's models do not assume that such an advantage leads to the elimination of the disproportional representation of language groups among employers and 


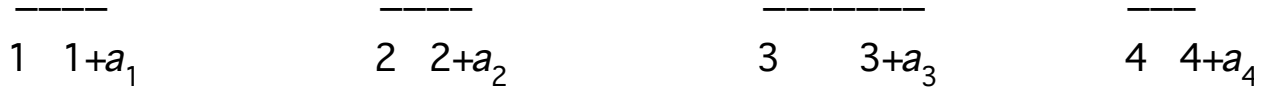

Figure 1. Partition of hypothetical population into native-language communities

a consequent elimination of the wage disparity. In addition, Lang's assumption of uniform learning costs precludes any prediction of an equilibrium in which at least some persons with each native language learn the other language. Yet we know that reciprocal language learning takes place, and it would be interesting to speculate about the assumptions that would predict an equilibrium that exhibits it.

In this paper we do not restrict ourselves to earnings as a mechanism or to firms as a milieu of the incentive to learn languages. We also abandon the two-language restriction imposed by both Grenier and Lang. Our aim is to predict some general features of distributions of nonnative language skills. We shall impose no limit on the number of languages in existence. We shall permit languages with native speakers and languages without native speakers to exist. The most important restriction from others' models that we keep intact is the treatment of the distribution of native languages as fixed.

Our model assumes that there is a positive number of languages in the world. At least one of these languages is natural, i.e. a language that has native speakers. There may also be any number of auxiliary languages, i.e. languages with no native speakers. Since everyone is assumed to have one and only one native language, the world population can be partitioned into communities, each community consisting of the native speakers of a particular language. We represent the members of the world population as a continuum of size 1 . We represent the $i$ th community as a continuum of size $a_{i}$. We choose to identify each member of the $i$ th community with a number $s$ such that $i \leq s \leq i+a_{i}$. Geometrically, the world population can be represented as a set of $n$ line segments, where $n$ is the number of communities. The $i$ th community is represented as the line segment from $i$ to $i+a_{i}$. Figure 1 gives an example of such a geometric representation for a world of four communities. The sum of the lengths of the four line segments is 1 .

We model this continuum as a continuum of players of a noncooperative normal-form game. Each player chooses what set of additional (non-native) languages to learn. We consider only pure strategies. Thus, if there are $m$ languages in the world, each player (represented as a point on one of the line segments) has a set of $2^{m-1}$ pure strategies among which to choose. A player in figure 1, for example, has eight possible pure strategies if there are no auxiliary languages, sixteen if there is one auxiliary language, and so forth.

The players' payoffs are the net benefits they derive from learning the languages that they learn. The positive term in a payoff is the player's communicative benefit. The negative term is the player's learning cost. A person's payoff is the difference between 
$2+t$

\begin{tabular}{|c|c|c|c|c|c|c|c|c|}
\hline \multirow[b]{2}{*}{ None } & \multicolumn{2}{|c|}{ None } & \multicolumn{2}{|c|}{1} & \multicolumn{2}{|c|}{3} & \multicolumn{2}{|c|}{$1 \& 3$} \\
\hline & 0 & 0 & $b_{12}(t)$ & $b_{21}(s)$ & 0 & 0 & $b_{12}(t)$ & $b_{21}(s)$ \\
\hline $1+s$ & $b_{12}(t)$ & $b_{21}(s)$ & $b_{12}(t)$ & $b_{21}(s)$ & $b_{12}{ }^{(t)}$ & $b_{21}(s)$ & $b_{12}(t)$ & $b_{21}(s)$ \\
\hline 3 & 0 & 0 & $b_{12}(t)$ & $b_{21}(s)$ & $b_{12}{ }^{(t)}$ & $b_{21}(s)$ & $b_{12}(t)$ & $b_{21}(s)$ \\
\hline $2 \& 3$ & $b_{12}(t)$ & $b_{21}(s)$ & $b_{12}(t)$ & $b_{21}(s)$ & $b_{12}(t)$ & $b_{21}(s)$ & $b_{12}(t)$ & $b_{21}(s)$ \\
\hline
\end{tabular}

Figure 2. Weight of two players in one another's communicative benefits

these: the communicative benefit minus the learning cost.

A player's communicative benefit is proportional to the importance-weighted fraction of the world population that shares at least one language with the player. Any language community has an importance function that assigns some importance to each member of the world population. To compute a player's communicative benefit, we use the importance function of the player's community to give a weight to everyone in the world with whom the person shares knowledge of at least one language. We give a weight of 0 to everyone with whom the person does not have a common language. The integral of the world population, thus weighted, is the player's communicative benefit.

To see how the communicative benefit is defined, consider a world with 2 native languages and one auxiliary language. Let $1+s$ be one of the players in community 1 , and $2+t$ be one of the players in community 2 . Suppose that community 1 's importance function assigns an importance of $b_{12}(t)$ to $2+t$, while community 2 's importance function assigns an importance of $b_{21}(s)$ to $1+s$. Then figure 2 shows the weight that each of these two players has in the other's communicative benefit, depending on the pair of strategies that they adopt. The rows are labeled with the four possible strategies for player $1+s$ (learn no additional language, learn language 2 , etc.), and the columns are labeled with the four possible strategies for player $2+t$. The left entry in each cell is the weight of player 2 $+t$ in player $1+s$ 's communicative benefit, and the right entry in each cell is the weight of player $1+s$ in player $2+t$ 's communicative benefit.

The importance function is intended to reflect the fact that economic, geographical, and other attributes of a person affect the value of being able to communicate with the person. When a person has unequal importance to several other persons, a typical reason is differences in geographical proximity. Since language communities tend to be geographically concentrated, we allow importance functions to differ among language communities but not within them. With this definition of communicative benefit, any two players who belong to the same community and adopt the same strategy must get the same communicative benefit. 
A player's learning cost is the product of two factors: a linguistic factor and a personal factor. The linguistic factor depends only on a person's languages: native and additional. Any two players who have the same native language and learn the same set of additional languages share the same linguistic factor. By assumption, the linguistic factor is always nonnegative, and it is 0 for any player who learns no additional language. These assumptions about the linguistic factor reflect three facts: (1) some languages are more difficult to learn than others, (2) the effort required in learning a combination of languages is not necessarily the sum of the efforts of learning the languages in the combination, and (3) the difficulty of learning a combination of languages depends on the native language of the learner.

The other component of a player's learning cost is a personal factor, which reflects the differences among language learners in aptitudes and motivations. The personal factor is a nonnegative number assigned to a player. For simplicity of analysis, it is assumed that within any language community no player has the same personal factor as any other player. Thus, any two players with the same native language who learn no additional language have the same learning cost (0), but if they learn the same additional language or languages they must have different learning costs. We have already assumed that their communicative benefit is identical. Therefore, with unequal learning costs they must have different payoffs.

Our first substantively interesting result describes a feature of the players' payoffs. The payoff to any player is a function of the strategy of that player and the strategies of all players who are members of other communities. So, a player's payoff is independent of the strategies of the other members of that player's own community. The reason is that a player's communicative benefit depends on the subset of the world population that shares at least one language with the player. But every member of the player's community shares a language with the player (their native language). This fact cannot be changed by any strategy that another member of this community adopts. Thus, the only way in which a player can affect the payoff of another player in the same community is indirectly: by affecting the choices of players in other communities. For example, in our model a native speaker of Japanese does not directly affect the payoff of another native speaker of Japanese. But, if one native speaker of Japanese learns English, this choice may reduce the incentive of a native speaker of English or some other language to learn Japanese, and the reduced learning of Japanese that results from this incentive reduction may affect the payoffs of other native speakers of Japanese.

Our analysis proceeds by examining the features of best replies and group best replies. A best reply is a strategy that maximizes the adopting player's payoff, given the combination of strategies adopted by all other players. The combination of the strategies adopted by all the members of a community is called a group strategy. If every player in a community adopts a best reply to the combination of all other players' strategies, then we 
say that the community's group strategy is a group best reply. Referring to group strategies and group best replies is only an analytical device, since the players in our game are individuals, not communities.

Our next result describes a regularity in best replies. Consider the members of one language community. For any combination of the other communities' members' strategies, each member of this community has at least one strategy that is a best reply. A strategy that is a best reply for one member may not be a best reply for some other member. We show, however, that whenever a strategy is a best reply for two members it is also a best reply for all members whose personal factors of their learning costs lie between those of the two members. In terms of a geometric representation, we can arrange the members of a community in order of increasing personal factors on the line segment that represents their community. If we do this, then any strategy that is a best reply at more than one point in a community is a best reply everywhere in some interval within the line segment, and nowhere else in the community. This result leads to an empirical prediction that, among the native speakers of any language, those who learn the same set of foreign languages will tend to be more similar in the extent of their (dis)inclination and (in)ability to learn languages than is true for any more loosely defined set of language learners. For example, the variance in scores on a language aptitude test should be greater among all those native speakers of German who have learned English and any one other foreign language than among the subsets of native German speakers who have learned English and French, who have learned English and Russian, etc.

We build on this result to show that any group best reply exhibits a negative association between the two factors of the learning cost. When a community adopts a group best reply, for any two members in the community whose learning costs have unequal linguistic factors, the one with the higher linguistic factor (the member who learns the more expensive set of languages) has the lower personal factor (finds it easier to learn languages). Suppose we arrange the members of a community from left to right in order of increasing personal factors, and suppose the community adopts a group best reply which involves more than one strategy. As we move from left to right, we find personal factors everywhere rising, while at each point where the linguistic factor changes it declines. An example of this pattern for a world with two natural languages and one auxiliary language is illustrated in figure 3 .

As we move from left to right in a community that adopts a group best reply, we must also find communicative benefit decreasing whenever there is an decrease in the linguistic factor. If this relationship did not exist, then the community would not be adopting a group best reply, because some member of the community could get a higher payoff by changing to a strategy with a lower linguistic factor.

As the linguistic factor decreases moving from left to right, not only do communicative benefits decrease, but so do payoffs. It might be imagined that this is not always true. 


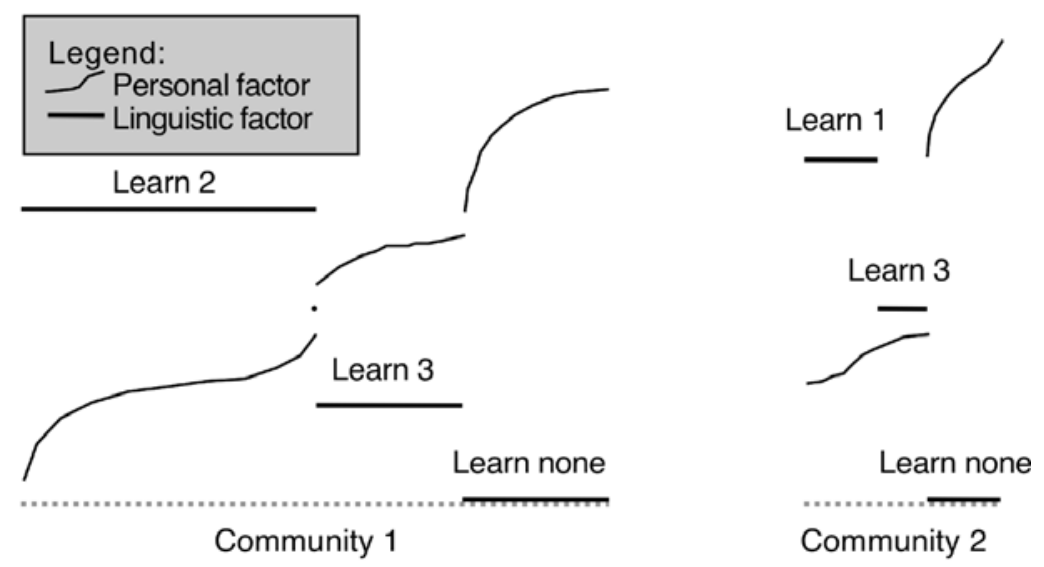

Figure 3. Illustration of group best replies in a two-community, threelanguage world

Under some conditions, the drop in the linguistic factor of the learning cost might be steep enough to counteract both the drop in the communicative benefit and the rise in the personal factor. But we show that there are no conditions that allow payoffs to rise or remain constant as the linguistic factor decreases. This result can be interpreted as saying that, in a community adopting a group best reply, those who learn more difficult sets of languages get higher profits, despite the difficulty of the languages they learn.

Having determined some features of best replies, we move on to show that it is possible for every player to adopt a best reply at the same time. In other words, at least one equilibrium point exists. There are several steps in proving this result.

First, we arbitrarily resolve any ties that may exist in the linguistic costs of the possible strategies for the players in any community. In other words, we label the possible strategies in a community with the numbers 1 to $2^{m-1}$, in order of decreasing linguistic cost. Whenever we encounter two or more strategies having the same linguistic cost, we number them in any order.

The next step is to construct a new game, whose players are the communities. In this new, or aggregated, game, we require the members of any community to adopt individual strategies that are numbered in the same order as the members' personal costs are ordered. Thus, if any two members of a community adopt different individual strategies, the member with the higher personal factor must adopt the higher-numbered strategy. This requirement limits the set of possible group strategies for the communities playing the aggregated game. A community must be partitioned into $2^{m-1}$ intervals, such that the $k$ th interval is the locus of members adopting the individual strategy numbered $k$. We limit the communities' strategy sets even further by defining a group strategy only as the set of 
upper bounds of these $2^{m-1}$ intervals. To see that this definition restricts the set of strategies, consider a point $x$ in a community and suppose that the members to the immediate left of $x$ adopt strategy $k$ and the members to the immediate right of $x$ adopt strategy $k+w$. Then the member precisely at point $x$ can adopt any of the strategies $k, k+$ $1, \ldots, k+w$. But it makes no difference to the group strategy what the individual strategy of this border member is, since the upper bounds of the intervals are unaffected. A community $i$ 's strategy in the aggregated game is thus a nondecreasing set of $2^{m-1}$ border points, each located between $i$ and $i+a_{i}$ and the last of them being equal to $i+a_{i}$.

The payoffs to the community players in the aggregated game are the respective integrals of the payoffs to their individual members in the original game. Each combination of strategies in the aggregated game induces a unique payoff to each community, despite the fact that the group strategies may leave the individual strategies of their border members incompletely specified. The reason is that the border members are only an infinitesimal fraction of all the members. The payoffs of the $2^{m-1}$ border members of a community (or of any finite number of its members) do not affect the integral of the payoffs of all the members of the community.

The last step in proving the existence of an equilibrium in the original game involves establishing relationships between the original and the aggregated games. We first show that whenever there is an equilibrium point in an aggregated game there is also an equilibrium point in the original game to which the aggregated game corresponds. We then show that for each aggregated game there is always at least one equilibrium point. But for each original game at least one corresponding aggregated game exists. These findings imply that there is always at least one equilibrium point in any original game.

In this paper we say nothing about the number and nature of the equilibria whose existence we prove. As an aid to intuitions, however, we picture one equilibrium in figure 4 . The example is a world with three language communities, whose shares of the population are $0.5,0.35$, and 0.15 , respectively. One auxiliary language exists, implying that there are four languages. For each player, three of the languages are open to choice, so each player has eight available strategies. The importance weight function of each community assigns identical weights to all players; thus, everyone is equally important for everyone else as a potential communication partner. The linguistic factors of the learning costs are additive functions of the languages contained in the learned sets of languages, with each natural language contributing 1.8 and the auxiliary language contributing 0.6 to the linguistic factor, regardless of the learner's native language. Finally, the personal factors of the learning cost are distributed uniformly in each community from 0 to 1 , shown as diagonal dashed lines in the figure.

In the equilibrium shown for this world in figure 4, only three of the eight possible strategies in each language community are chosen. These are (1) to learn both nonnative natural languages, (2) to learn the nonnative natural language with the larger number of 


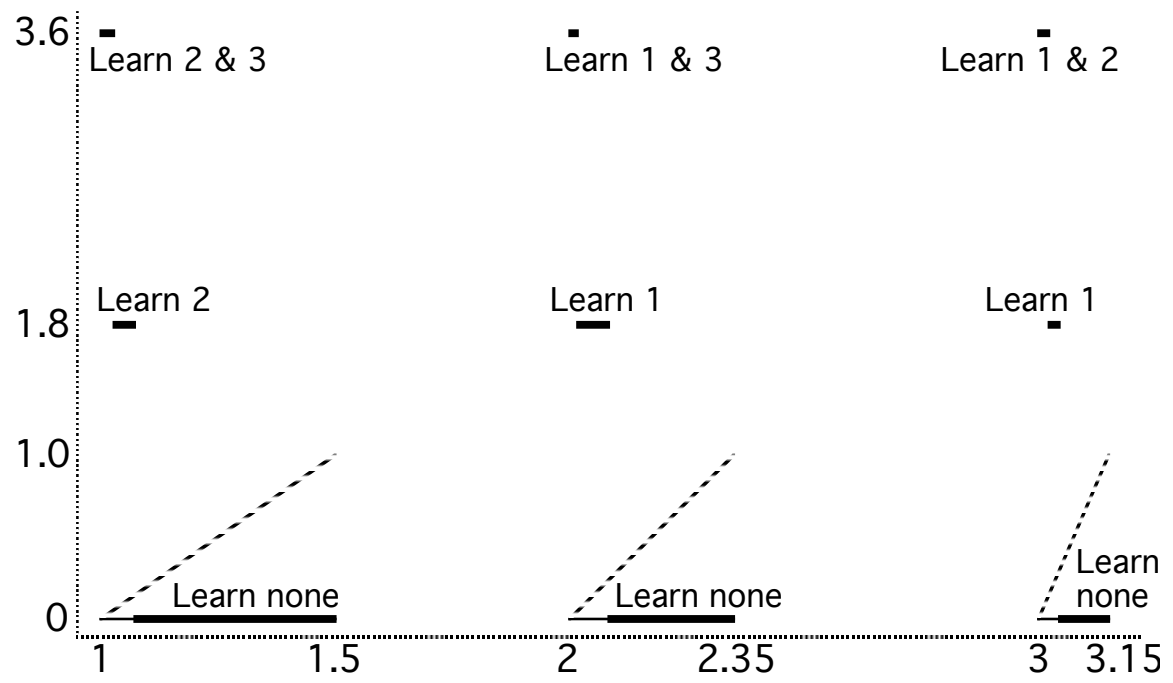

Figure 4. Illustration of equilibrium in a three-community, four-language world

native speakers, or (3) to learn nothing. The players are arranged in order of increasing personal factors of learning cost, with this personal factor plotted by dashed lines. When the players are so arranged, each community is partitioned into three convex sets of players, all players in the $i$ th set choosing the $i$ th strategy. The boundaries between the adjacent sets of players are, in distance from the community's origin, 0.0292 and 0.0729 in community $1,0.0205$ and 0.0875 in community 2 , and 0.0219 and 0.0448 in community 3. The sets of players choosing these strategies are plotted at heights equal to the linguistic factors of their learning costs.

In this example, the proportion of each community that learns two nonnative languages is largest in the smallest community and smallest in the largest community. The same is true for the proportion that learns at least one nonnative language. We also notice that no player learns the auxiliary language. These results match well-known tendencies: those who learn nonnative languages tend to choose the most widely spoken ones; members of small language communities are more likely to learn foreign languages than members of large communities; and few learn auxiliary languages, despite their relatively low learning costs.

Another insight we may derive from our results concerns the phenomenon of apparently deliberate foreign-language incompetence. It has been claimed that the native speakers of certain languages (such as English and French) succeed in getting the native speakers of other languages to learn their languages. The strategy allegedly used to attain this effect is the intentional neglect of foreign-language learning and the ostentatious exhibition of low language-learning aptitudes. In the terms of our model, this claim can be rephrased into a suggestion that the native speakers of a language benefit from having high personal factors 
and hence high learning costs. The predictions from our model do not support this idea. Our predictions are compatible with the possibility that a player's payoff can be an increasing function of the learning costs of the player's fellow native speakers. But payoffs to a member of a community cannot, in any equilibrium, be an increasing function of the member's learning cost. Thus, even if the language community with the highest payoffs is the one whose members have the greatest average difficulty learning foreign languages, it is the easy learners within this group who have the highest payoffs in their group.

\section{The model}

The model describes a world with $m$ languages, $1, \ldots, m$. Every individual has one and only one of these languages as his native language. He is a native speaker of that language. Every language other than an individual's native language is a foreign language for that individual. Some languages may be foreign to all individuals. Languages with native speakers will be called natural languages, and the others will be referred to as auxiliary languages. Let languages $1, \ldots, n$, with $n \leq m$, be the natural languages. Let $M$ be the set $\{1, \ldots, m\}$ of all languages, and let $N$ be the set $\{1, \ldots, n\}$ of all natural languages.

Players and strategies. The set of all individuals with native language $i$ is the language community $i$ or, for short, community $i$. Each language community $i$ is described as a continuum of individuals represented as points in an interval $\left[i, i+a_{i}\right]$, with $a_{i}>0$. The measure of all individuals is normalized to 1 :

$$
\sum_{i=1}^{n} a_{i}=1 .
$$

We call $a_{i}$ the size of language community $i$. Our model may be looked upon as a game with a continuum of players. The players are the members of the language communities 1 , $\ldots, n$. The pure strategies are possible sets $C$ of languages to be learned. Of course, a member of language community $i$ always learns language $i$ and has no choice in this respect. The set of all pure strategies $C$ of a member $i+s$ of community $i$ is as follows:

$$
M_{i}=\{C \mid i \in C \subseteq M\}
$$

A group strategy of community $i$ is a Borel measurable function which assigns a pure strategy $\varphi_{i}(s)$ to every member $i+s$ of community $i$ :

$$
\varphi_{i}:\left[0, a_{i}\right] \rightarrow M_{i}
$$

It is notationally convenient to define $\varphi_{i}$ on $\left[0, a_{i}\right]$ rather than on $\left[i, i+a_{i}\right]$. The set of all group strategies of community $i$ is denoted by $\Phi_{i}$. A strategy combination 


$$
\varphi=\left(\varphi_{1}, \ldots, \varphi_{n}\right)
$$

is an $n$-tuple of group strategies $\varphi_{i} \in \Phi_{i}$. The set of all strategy combinations is denoted by $\Phi$.

An $i$-incomplete strategy combination $\varphi_{-i}$ is an $(n-1)$-tuple of group strategies $\varphi_{j} \in \Phi_{j}$ with $j \neq i$ :

$$
\varphi_{-i}=\left(\varphi_{1}, \ldots, \varphi_{i-1}, \varphi_{i+1}, \ldots, \varphi_{n}\right) .
$$

The set of all $i$-incomplete strategy combinations is denoted by $\Phi_{-i}$. The $i$-incomplete strategy combination $\varphi_{-i}$ whose components agree with the corresponding components of a strategy combination $\varphi$ is called the $i$-incomplete combination in $\varphi$.

Learning costs. There are two influences on a player's payoff: "communicative benefit" and "learning cost". We first turn our attention to learning cost. Consider a member $i+s$ of community $i$ who chooses $C$. His learning cost $K_{i+s}(C)$ is modeled as follows:

$$
K_{i+s}(C)=f_{i}(s) g_{i}(C),
$$

where $f_{i}$ is a bounded monotonically increasing nonnegative function defined on $\left[0, a_{i}\right]$ and $g_{i}$ is a function defined on $M_{i}$ with

$$
g_{i}(C) \geq 0 \text { for all } C \in M_{i}
$$

and

$$
g_{i}(\{i\})=0
$$

Learning costs are the product of a personal factor $f_{i}(s)$ and a linguistic factor $g_{i}(C)$. The personal factor summarizes influences like aptitude. We think of the members of community $i$ as arranged according to their personal factors. Therefore the monotonicity assumption on $f_{i}$ is not very restrictive.

Communicative benefit. We now turn our attention to the communicative benefit of language learning. Every other individual $j+t$ with whom a player $i+s$ has a common language provides a communication opportunity, which contributes to the communicative benefit of player $i+s$. The importance of the opportunity to communicate with $j+t$ may depend on various characteristics of $j+t$ such as income or geographical distance. Therefore, in the computation of communicative benefit, communicative opportunities are weighted by an importance factor $b_{i j}(t)$. In order to express the communicative benefit we make use of the following notation. For any two subsets $C$ and $D$ of $M$ define:

$$
\delta(C, D)=\left\{\begin{array}{l}
1 \text { for } C \cap D \neq \varnothing \\
0 \text { for } C \cap D=\varnothing
\end{array} .\right.
$$


Let $\varphi=\left(\varphi_{1}, \ldots, \varphi_{n}\right)$ be a strategy combination. The communicative benefit $U_{i}(C, \varphi)$ obtained by a member of community $i$ from $C$ if $\varphi$ is played by all other individuals is defined as follows:

$$
U_{i}(C, \varphi)=\sum_{j=1}^{n} \int_{0}^{a_{j}} b_{i j}(t) \delta\left(C, \varphi_{j}(t)\right) d t .
$$

It is assumed that for $i=1, \ldots, n$ and $j=1, \ldots, n$ the importance weight functions

$$
b_{i j}:\left[0, a_{j}\right] \rightarrow \mathbb{R}_{+}
$$

are bounded, nonnegative and piecewise continuous with finitely many discontinuities. This assumption together with the measurability of the $\varphi_{j}$ guarantees the existence of the integrals on the right-hand side of (10).

Since all $C \in M_{i}$ contain $i$, we have:

$$
U_{i}(C, \varphi)=\int_{0}^{a_{i}} b_{i i}(t) d t+\sum_{\substack{j=1 \\ j \neq i}}^{n} \int_{0}^{a_{j}} b_{i j}(t) \delta\left(C, \varphi_{j}(t)\right) d t .
$$

This shows that $U_{i}(C, \varphi)$ does not depend on $\varphi_{i}$, but only on the $i$-incomplete combination $\varphi_{-i}$ in $\varphi$. Accordingly, we shall also use the notation $U_{i}\left(C, \varphi_{-i}\right)$ :

$$
U_{i}\left(C, \varphi_{-i}\right)=U_{i}(C, \varphi) \text { with } \varphi_{-i} \text { in } \varphi
$$

Payoffs. We now define the payoff $H_{i+s}\left(C, \varphi_{-i}\right)$ to a member $i+s$ of community $i$ obtained against an $i$-incomplete strategy combination $\varphi_{-i} \in \Phi_{-i}$ for $C \in M_{i}$ :

$$
H_{i+s}\left(C, \varphi_{-i}\right)=U_{i}\left(C, \varphi_{-i}\right)-f_{i}(s) g_{i}(C)
$$

for $i=1, \ldots, n$ and $s \in\left[0, a_{i}\right]$. The payoff function $H$ assigns $H_{i+s}\left(C, \varphi_{-i}\right)$ to every triple $(i+$ $s, C, \varphi_{-i}$ ), where $i+s$ is a member of community $i$, where $C$ is one of his pure strategies, and where $\varphi_{-i}$ is an $i$-incomplete strategy combination. The pure strategy sets $M_{i}$ of the members of the language communities together with $H$ constitute a game in normal form with a continuum of players. The measurability requirement imposes a restriction on the region where payoffs are defined, but apart from this a game in normal form with a continuum of players is a straightforward modification of the usual concept of a game in normal form.

Summary of assumptions. Let $a=\left(a_{1}, \ldots, a_{n}\right)$ be the vector of the $a_{i}$ and let $f=\left(f_{1}\right.$, $\left.\ldots, f_{n}\right)$ and $g=\left(g_{1}, \ldots, g_{n}\right)$ be the vectors of the functions $f_{i}$ and $g_{i}$, respectively. Moreover, let $b$ be the $n, n$ matrix of the functions $b_{i j}$ with $i=1, \ldots, n$ and $j=1, \ldots, n$. The sextuple $(m$, $n, a, b, f, g)$ summarizes the elements which have to be fixed in a specification of the model. The notation $\Gamma(m, n, a, b, f, g)$ indicates the normal form game resulting from the 
specification $(m, n, a, b, f, g)$. In the following we shall give a summary of the assumptions made about the elements to be specified.

1. $m$, the number of languages, and $n$, the number of natural languages, are positive integers with $m \geq n$.

2. The components $a_{i}$ of $a$, the sizes of language communities, are positive and sum to 1 .

3. For $i=1, \ldots, n$ and $j=1, \ldots, n$, the importance weight functions $b_{i j}$ are bounded, nonnegative, and piecewise continuous with finitely many discontinuities.

4. For $i=1, \ldots, n$, the personal factor $f_{i}(s)$ of the learning cost is a bounded monotonically increasing nonnegative function.

5. For $i=1, \ldots, n$, the linguistic factor $g_{i}$ of the learning cost has the properties $g_{i}(\{i\})=$ 0 and $g_{i}(C) \geq 0$ for all $C \in M_{i}$.

\section{Equilibrium points and the structure of group best replies}

The model will be analyzed as a noncooperative game. In this section we shall prepare for the investigation of equilibrium points in pure strategies. Definitions and statements will refer to an arbitrarily fixed game $\Gamma(m, n, a, b, f, g)$.

Best replies. Loosely speaking, a best reply is a strategy which maximizes the payoff of a player given the behavior of all others. The payoff that $i+s$ obtains by a pure strategy $C \in M_{i}$ depends only on $\varphi_{i}(s)$ and $\varphi_{-i}$. Therefore, player $i+s$ does not have to know $\varphi_{i}(t)$ for $t \neq s$ in order to determine his best reply. Let $\varphi_{-i}$ be an $i$-incomplete strategy combination. Formally, $D \in M_{i}$ is a best reply of $i+s$ to $\varphi_{-i}$ if we have:

$$
H_{i+s}\left(D, \varphi_{-i}\right)=\max _{C \in M_{i}} H_{i+s}\left(C, \varphi_{-i}\right) \text {. }
$$

We say that $D$ is a best reply to $\varphi=\left(\varphi_{1}, \ldots, \varphi_{n}\right)$ if $D$ is a best reply to the $i$-incomplete strategy combination $\varphi_{-i}$ in $\varphi$.

A group strategy $\psi_{i} \in \Phi_{i}$ is a group best reply or simply a best reply to an $i$-incomplete combination $\varphi_{-i}$ if for every member $i+s$ of community $i$ his strategy $\psi_{i}(s)$ is a best reply to $\varphi_{-i}$. We say that $\psi_{i}$ is a group best reply or simply a best reply to $\varphi=\left(\varphi_{1}, \ldots, \varphi_{n}\right)$ if $\psi_{i}$ is a best reply to the $i$-incomplete strategy combination $\varphi_{-i}$ in $\varphi$. A strategy combination $\psi=$ $\left(\psi_{1}, \ldots, \psi_{n}\right)$ is a best reply combination for $\varphi=\left(\varphi_{1}, \ldots, \varphi_{n}\right)$ or simply a best reply to $\varphi$ if for $i$ $=1, \ldots, n$ the group strategy $\psi_{i}$ is a best reply to $\varphi$.

A group strategy $\psi_{i}$ is an almost best reply to an $i$-incomplete strategy combination $\varphi_{-i}$ 
or a strategy combination $\varphi$ if for $0 \leq t \leq a_{i}$ with the exception of finitely many values of $t$ the strategy $\psi_{i}(t)$ is a best reply of player $i+t$ to $\varphi_{-i}$ or $\varphi$, respectively.

Equilibrium points. Since mixed strategies are not considered in this paper, the term "equilibrium point" will always refer to an equilibrium point in pure strategies. A strategy combination $\varphi=\left(\varphi_{1}, \ldots, \varphi_{n}\right)$ is an equilibrium point if $\varphi$ is a best reply to itself or, in other words, if for $i=1, \ldots, n$ the group strategy $\varphi_{i}$ is a best reply to $\varphi_{-i}$ in $\varphi$. Analogously, an almost-equilibrium point is a strategy combination which is an almost best reply to itself.

Lemma 1. Let $\varphi=\left(\varphi_{1}, \ldots, \varphi_{n}\right)$ be an almost-equilibrium point and let $\psi=\left(\psi_{1}, \ldots, \psi_{n}\right)$ be a strategy combination such that, for $i=1, \ldots, n$ and $0 \leq t \leq a_{i}$, wherever $\varphi_{i}(t)$ is a best reply of $i+t$ to $\varphi$ the strategy $\psi_{i}(t)$ agrees with $\varphi_{i}(t)$, and wherever $\varphi_{i}(t)$ is not a best reply of $i+t$ to $\varphi$ the strategy $\psi_{i}(t)$ is a best reply of $i+t$ to $\varphi$. Then $\psi$ is an equilibrium point.

Proof. The transition from $\varphi$ to $\psi$ involves only a finite number of changes. Therefore, we have

$$
H_{i+t}\left(C, \psi_{-i}\right)=H_{i+t}\left(C, \varphi_{-i}\right)
$$

for $i=1, \ldots, n$, for $0 \leq t \leq a_{i}$, and for every $C \in M_{i}$, with $\varphi_{-i}$ in $\varphi$ and $\psi_{-i}$ in $\psi$.

Consequently, the assertion holds.

Comment. Lemma 1 shows how an almost-equilibrium point can be changed into an equilibrium point by the adjustment of the strategies of finitely many players. In order to prove the existence of equilibrium points and to explore their structural properties, it is sufficient to investigate almost-equilibrium points.

We shall now derive a useful result on the set of the members of a language community $i$ for whom a given strategy $C$ is a best reply to an $i$-incomplete strategy combination $\varphi_{-i}$. Then we shall prove a theorem which exhibits an interesting structural feature of group best replies: those who learn more difficult sets of languages have lower personal learning costs.

Lemma 2. Let $\varphi_{-i}$ be an $i$-incomplete strategy combination and let $C \in M_{i}$ be a strategy for a member of language community $i$. Moreover, let $I$ be the set of all $t$ with $0 \leq t$ $\leq a_{i}$ such that $C$ is a best reply of $i+t$ to $\varphi_{-i}$. If the set $I$ contains at least two points, then $I$ is a closed, open, or half-open interval.

Proof. Assume that $s$ and $t$ with $s \neq t$ belong to $I$. For every $D \in M_{i}$ with $D \neq C$ we have:

$$
\begin{aligned}
& U_{i}\left(C, \varphi_{-i}\right)-f_{i}(s) g_{i}(C) \geq U_{i}\left(D, \varphi_{-i}\right)-f_{i}(s) g_{i}(D) ; \\
& U_{i}\left(C, \varphi_{-i}\right)-f_{i}(t) g_{i}(C) \geq U_{i}\left(D, \varphi_{-i}\right)-f_{i}(t) g_{i}(D) .
\end{aligned}
$$


For every $t^{\prime}$ with $s<t^{\prime}<t$, the personal cost factor $f_{i}\left(t^{\prime}\right)$ is a convex linear combination of $f_{i}(s)$ and $f_{i}(t)$; in other words, for some $\alpha$ with $0<\alpha<1$ the following is true:

$$
f_{i}\left(t^{\prime}\right)=(1-\alpha) f_{i}(s)+\alpha f_{i}(t) .
$$

The sum of (17) multiplied by $(1-\alpha)$ and (18) multiplied by $\alpha$ yields

$$
U_{i}\left(C, \varphi_{-i}\right)-f_{i}\left(t^{\prime}\right) g_{i}(C) \geq U_{i}\left(D, \varphi_{-i}\right)-f_{i}\left(t^{\prime}\right) g_{i}(D) .
$$

Consequently, $C$ is a best reply of $i+t^{\prime}$ to $\varphi_{-i}$ for every $t^{\prime}$ with $s<t^{\prime}<t$. It follows that the assertion of the lemma holds.

Theorem 1 (on group best replies). Let $\varphi_{-i}$ be an $i$-incomplete strategy combination and let $\varphi_{i}$ be a group best reply to $\varphi_{-i}$. Moreover, let $i+s$ and $i+t$ be two members of community $i$ with

$$
g_{i}\left(\varphi_{i}(s)\right)>g_{i}\left(\varphi_{i}(t)\right) .
$$

Then we have:

$$
s<t .
$$

Proof. The following inequalities are consequences of the fact that $\varphi_{i}(s)$ and $\varphi_{i}(t)$ are best replies to $\varphi_{-i}$ :

$$
\begin{aligned}
& U_{i}\left(\varphi_{i}(s), \varphi_{-i}\right)-f_{i}(s) g_{i}\left(\varphi_{i}(s)\right) \geq U_{i}\left(\varphi_{i}(t), \varphi_{-i}\right)-f_{i}(s) g_{i}\left(\varphi_{i}(t)\right) ; \\
& U_{i}\left(\varphi_{i}(s), \varphi_{-i}\right)-f_{i}(t) g_{i}\left(\varphi_{i}(s)\right) \leq U_{i}\left(\varphi_{i}(t), \varphi_{-i}\right)-f_{i}(t) g_{i}\left(\varphi_{i}(t)\right) .
\end{aligned}
$$

Subtraction of (24) from (23) yields

$$
\left[f_{i}(t)-f_{i}(s)\right] g_{i}\left(\varphi_{i}(s)\right) \geq\left[f_{i}(t)-f_{i}(s)\right] g_{i}\left(\varphi_{i}(t)\right)
$$

or, equivalently,

$$
\left[f_{i}(t)-f_{i}(s)\right]\left[g_{i}\left(\varphi_{i}(s)\right)-g_{i}\left(\varphi_{i}(t)\right)\right] \geq 0 .
$$

In view of (21), the second factor on the left hand side of (26) is positive. Therefore, the first factor must be nonnegative, giving us:

$$
f_{i}(s) \leq f_{i}(t)
$$

Since $\varphi_{i}(s)$ and $\varphi_{i}(t)$ must be different, we have $s \neq t$. Moreover, $f_{i}$ is monotonically increasing. Consequently, (22) holds.

Remark. It can be seen immediately that the theorem has the following consequence: $s<t$ implies $g_{i}\left(\varphi_{i}(s)\right) \geq g_{i}\left(\varphi_{i}(t)\right)$. Moreover, it follows with the help of (23) that $g_{i}\left(\varphi_{i}(s)\right)>$ $g_{i}\left(\varphi_{i}(t)\right)$ implies 


$$
U_{i}\left(\varphi_{i}(s), \varphi_{-i}\right)>U_{i}\left(\varphi_{i}(t), \varphi_{-i}\right) .
$$

It is also worth pointing out that we have

$$
H_{i+s}\left(\varphi_{i}(s), \varphi_{-i}\right)>H_{i+t}\left(\varphi_{i}(t), \varphi_{-i}\right)
$$

for $s<t$ and $g_{i}\left(\varphi_{i}(t)\right)>0$, since under these conditions $i+s$ can obtain a higher payoff than $i$ $+t$ by choosing the same strategy, $\varphi_{i}(t)$.

\section{Monotone group strategies and aggregated games}

Theorem 1 shows that group best replies have the property that the two factors of learning costs are inversely connected. Higher personal factors are connected with lower or equal linguistic factors. Loosely speaking, we may say that a group best reply must respect the order of decreasing linguistic factors. The order of decreasing linguistic factors may be looked upon as a ranking of the individual strategies for the members of a language community. In the presence of equal linguistic factors for different individual strategies, this ranking has ties. For every possible strengthened order that one can create by arbitrarily breaking all ties, we shall define a narrower "monotonicity class" of group strategies which respect this strengthened order of decreasing linguistic factors. We shall prove that every monotonicity class contains a group best reply to every $i$-incomplete strategy combination. This result will be the basis of our construction of "aggregated games".

Within a monotonicity class a group strategy is almost completely characterized by the border points of the intervals where the same individual strategy is used. These border points form a "border point vector". Within the monotonicity class the group strategies with the same border point vector form a "border equivalence class". Two members of the same border equivalence class differ at most at finitely many points, namely the border points.

In an aggregated game, the players are the language communities. Payoffs are "group payoffs" defined as integrals over individual payoffs. Strategies correspond to border equivalence classes within a particular monotonicity class. Formally, strategies are border point vectors.

In the presence of equal linguistic factors for different strategies for the individuals in a language community, more than one aggregated game exists. For every way of selecting a monotonicity class for each player (i.e. for each language community), we obtain a different aggregated game.

An equilibrium point of the original game is associated with every equilibrium point of every aggregated game. Therefore, the task of proving the existence of equilibrium points 
can be shifted from the original game to the aggregated games.

We continue to use the definitions and notations introduced in the previous sections. All statements refer to an arbitrarily selected fixed game $\Gamma(m, n, a, b, f, g)$ whose specification satisfies assumptions 1 to 5 .

Monotonicity classes. A member of community $i$ can learn up to $m-1$ foreign languages. Therefore, the number of pure strategies in the set $M_{i}$ is $2^{m-1}$. Let $r_{i}$ be a function which assigns one of the numbers $1, \ldots, 2^{m-1}$ to every $C \in M_{i}$, a different one for each $C \in M_{i}$. We call $r_{i}$ a ranking over $M_{i}$. We say that $r_{i}$ is cost compatible if, for every pair of strategies $C$ and $D$ in $M_{i}$, we have:

$$
r_{i}(C)<r_{i}(D) \text { for } g_{i}(C)>g_{i}(D) .
$$

Let $r_{i}$ be a cost compatible ranking over $M_{i}$. We say that a group strategy $\varphi_{i} \in \Phi_{i}$ is monotone with respect to $r_{i}$ if for $s$ and $t$ in $\left[0, a_{i}\right]$ the following is always true:

$$
r_{i}\left(\varphi_{i}(s)\right) \leq r_{i}\left(\varphi_{i}(t)\right) \text { for } s<t .
$$

The set of all group strategies $\varphi_{i} \in \Phi_{i}$ which are monotone with respect to $r_{i}$ is denoted by $\Psi_{i}\left(r_{i}\right)$. We call $\Psi_{i}\left(r_{i}\right)$ the monotonicity class of $r_{i}$.

Lemma 3. Let $\Psi_{i}\left(r_{i}\right)$ be the monotonicity class of a cost compatible ranking $r_{i}$ over $M_{i}$. Then $\Psi_{i}\left(r_{i}\right)$ contains a group best reply $\varphi_{i}$ to every $i$-incomplete strategy combination $\varphi_{-i}$.

Proof. For every $t$ with $0 \leq t \leq a_{i}$, let $\varphi_{i}(t)$ be that best reply $C$ of $i+t$ to $\varphi_{-i}$ which has the lowest rank $r_{i}(C)$ among all best replies of $i+t$ to $\varphi_{-i}$. Obviously, $\varphi_{i}$ is a group best reply to $\varphi_{-i}$. We have to show that $\varphi_{i}$ is monotone with respect to $r_{i}$. In view of theorem 1 , inequality (31) holds if $g_{i}\left(\varphi_{i}(s)\right)$ is different from $g_{i}\left(\varphi_{i}(t)\right)$. Suppose that $g_{i}\left(\varphi_{i}(s)\right)=$ $g_{i}\left(\varphi_{i}(t)\right)$, that $s<t$, and that (31) does not hold. Then $r_{i}\left(\varphi_{i}(s)\right)$ is greater than $r_{i}\left(\varphi_{i}(t)\right)$. Since $\varphi_{i}(s)$ is the lowest ranking best reply of $i+s$ to $\varphi_{-i}$, it follows that $\varphi_{i}(t)$ is not a best reply of $i+s$ to $\varphi_{-i}$. Therefore, we must have:

$$
U_{i}\left(\varphi_{i}(s), \varphi_{-i}\right)-f_{i}(s) g_{i}\left(\varphi_{i}(s)\right)>U_{i}\left(\varphi_{i}(t), \varphi_{-i}\right)-f_{i}(s) g_{i}\left(\varphi_{i}(t) .\right.
$$

In view of the fact that $g_{i}\left(\varphi_{i}(s)\right)$ and $g_{i}\left(\varphi_{i}(t)\right)$ are equal, we can conclude:

$$
U_{i}\left(\varphi_{i}(s), \varphi_{-i}\right)>U_{i}\left(\varphi_{i}(t), \varphi_{-i}\right) .
$$

It follows by (29) and the equality of $g_{i}\left(\varphi_{i}(s)\right)$ and $g_{i}\left(\varphi_{i}(t)\right)$ that $\varphi_{i}(t)$ cannot be a best reply of $i+t$ to $\varphi_{-i}$. This is a contradiction. Therefore, the assertion of the lemma is true.

Border point vectors. In the following we shall look at a fixed cost compatible ranking $r_{i}$. For $k=1, \ldots, 2^{m-1}$, the strategy $C \in M_{i}$ with $r_{i}(C)=k$ is denoted by $C_{i k}$. For every group strategy $\varphi_{i}$ in the monotonicity class $\Phi_{i}\left(r_{i}\right)$ of $r_{i}$, let $I_{i k}$ be the inverse image $\varphi_{i}^{-1}\left(C_{i k}\right)$ of $C_{i k}$ with respect to $\varphi_{i}$ or in other words the set of all $t \in\left[0, a_{i}\right]$ with $r_{i}\left(\varphi_{i}(t)\right)=k$. We use the 
notation $z_{i k}$ for the upper border point of $I_{i k}$; moreover, we define $z_{i 0}=0$. Of course, several border points $z_{i k}$ may coincide. Obviously, $I_{i k}$ is a closed, open, or half open interval between the border points $z_{i(k-1)}$ and $z_{i k}$. For $k=2^{m^{i k}} 1$, we always have $z_{i k}=a_{i}$. The vector

$$
z_{i}=\left(z_{i 0}, \ldots, z_{i 2^{m 1}}\right)
$$

is called the border point vector of $\varphi_{i}$. A border point vector always satisfies the following condition:

$$
0=z_{i 0} \leq z_{i 1} \leq \ldots \leq z_{i 2^{m-1}}=a_{i} .
$$

The set of all vectors $z_{i}$ of the form (34) with (35) is denoted by $Z_{i}$. We call $Z_{i}$ the border point vector set of language community $i$. The set of all group strategies $\varphi_{i} \in \Psi_{i}\left(r_{i}\right)$ which have the same border point vector $z_{i}$ is denoted by $\Lambda_{i}\left(z_{i}\right)$. We call $\Lambda_{i}\left(z_{i}\right)$ the border equivalence class of $z_{i}$. We say that two group strategies in $\Psi_{i}\left(r_{i}\right)$ are border equivalent if they are in the same border equivalence class. If $z_{i}=\left(z_{i 0}, \ldots, z_{i 2^{m-1}}\right)$ is the border point vector of a group strategy $\varphi_{i} \in \Psi_{i}\left(r_{i}\right)$, then we have:

$$
\varphi_{i}(t)=C_{i k} \text { for } z_{i(k-1)}<t<z_{i k}
$$

for $k=1, \ldots, 2^{m-1}$. Obviously, two border equivalent group strategies in $\Psi_{i}\left(r_{i}\right)$ can differ only at the border points of their common border point vector.

Combinations of rankings. A combination $r=\left(r_{1}, \ldots, r_{n}\right)$ of cost compatible rankings contains a cost compatible ranking $r_{i}$ for each language community $i=1, \ldots, n$. An $i$-incomplete combination $r_{-i}$ of cost compatible rankings contains a cost compatible ranking for every language community $j$ with $j \neq i$. We say than $r_{-i}$ is in $r$ if the components of $r_{-i}$ are the corresponding components of $r$. The set of all cost compatible rankings of language community $i$ is denoted by $R_{i}$; the symbol $R$ is used for the set of all combinations $r$ of cost compatible rankings, and $R_{-i}$ is the set of all $i$-incomplete combinations $r_{-i}$ of cost compatible rankings.

Border point systems. In the following we shall look at a fixed combination $r=\left(r_{1}\right.$, $\ldots, r_{n}$ ) of cost compatible rankings and the $i$-incomplete combination $r_{-i}$ in $r$. The monotonicity class $\Psi(r)$ of $r$ is the set of all strategy combinations $\varphi=\left(\varphi_{1}, \ldots, \varphi_{n}\right)$ with $\varphi_{i} \in$ $\Psi_{i}\left(r_{i}\right)$ for $i=1, \ldots, n$. Analogously, the monotonicity class $\Psi_{-i}\left(r_{-i}\right)$ of $r_{-i}$ is the set of all $i$ incomplete strategy combinations $\varphi_{-i}$ whose components $\varphi_{j}$ are in the corresponding monotonicity classes $\Psi_{j}\left(r_{j}\right)$. Let $\varphi=\left(\varphi_{1}, \ldots, \varphi_{n}\right)$ be a strategy combination in $\Psi(r)$. For $i=$ $1, \ldots, n$, let $z_{i}$ be the border point vector of $\varphi_{i}$ defined above. We call

$$
z=\left(z_{1}, \ldots, z_{n}\right)
$$

the border point system of $\varphi$. Analogously, the $i$-incomplete border point system $z_{-i}$ of an $i$ incomplete strategy combination $\varphi_{-i} \in \Psi_{-i}\left(r_{-i}\right)$ contains the border point vectors of the components of $\varphi_{-i}$. The set of all systems $z$ of the form (37) with $z_{i} \in Z_{i}$ is denoted by $Z$, and the set of all $i$-incomplete border point systems 


$$
z_{-i}=\left(z_{1}, \ldots, z_{i-1}, z_{i+1}, \ldots, z_{n}\right)
$$

with $z_{j} \in Z_{j}$ for all components $z_{j}$ of $z_{-i}$ is denoted by $Z_{-i}$. The border equivalence class $\Lambda(z)$ of $z=\left(z_{1}, \ldots, z_{n}\right)$ is the set of all strategy combinations $\varphi \in \Psi(r)$ whose border point system is $z$. Similarly, the border equivalence class $\Lambda_{-i}\left(z_{-i}\right)$ of an $i$-incomplete border point system $z_{-i}$ is the set of all $i$-incomplete strategy combinations $\varphi_{-i}$ whose $i$-incomplete border point system is $z_{-i}$. Two border point systems or two $i$-incomplete border point systems are border equivalent if they belong to the same border equivalence class.

Group payoffs. We continue to look at a fixed combination of cost compatible rankings $r=\left(r_{1}, \ldots, r_{n}\right)$. Let $\varphi=\left(\varphi_{1}, \ldots, \varphi_{n}\right)$ be a strategy combination in the monotonicity class $\Psi(r)$, and let $z=\left(z_{1}, \ldots, z_{n}\right)$ be the border point system of $\varphi$. The group payoff of a language community $i$ for $\varphi$ is defined as the integral over all individual payoffs for members of the language community. As we shall see, this group payoff is a function of $z$. The finitely many individual strategies at border points do not matter for the integral. Therefore, we introduce the following definition of the group payoff $P_{i}(z)$ of language community $i$ for $z$ :

$$
P_{i}(z)=\int_{0}^{a_{i}} H_{i+t}\left(\varphi_{i}(t), \varphi_{-i}\right) d t
$$

It follows by (12) and (13) that communicative benefits $U_{i}\left(C, \varphi_{-i}\right)$ can be expressed as follows:

$$
U_{i}\left(C, \varphi_{-i}\right)=\sum_{j=1}^{n} \sum_{k=1}^{2^{m-1}} \int_{z_{j(k-1)}}^{z_{j k}} b_{i j}(t) \delta\left(C, C_{j k}\right) d t
$$

Define:

$$
B_{i j}(s)=\int_{0}^{s} b_{i j}(t) d t
$$

With this notation, (40) assumes the following form:

$$
U_{i}\left(C, \varphi_{-i}\right)=\sum_{j=1}^{n} \sum_{k=1}^{2^{m-1}}\left[B_{i j}\left(z_{j k}\right)-B_{i j}\left(z_{j(k-1)}\right)\right] \delta\left(C, C_{j k}\right) .
$$

For $j=i$, the inner sum is nothing other than $B_{i i}\left(a_{i}\right)$, since we always have $\delta\left(C, C_{i k}\right)=1$. Therefore, the right hand side of (42) is a function of $z_{-i}$ in $z$. We introduce the symbol $U_{i}\left(C, z_{-i}\right)$ for the right hand side of (42): 


$$
U_{i}\left(C, z_{-i}\right)=B_{i i}\left(a_{i}\right)+\sum_{\substack{j=1 \\ j \neq i}}^{n} \sum_{k=1}^{2}\left[B_{i j}\left(z_{j k}\right)-B_{i j}\left(z_{j(k-1)}\right)\right] \delta\left(C, C_{j k}\right) .
$$

For $i=1, \ldots, n$, define

$$
F_{i}(s)=\int_{0}^{s} f_{i}(t) d t
$$

and

$$
L_{i}\left(z_{i}\right)=\sum_{k=1}^{2^{m-1}}\left[F_{i}\left(z_{i k}\right)-F_{i}\left(z_{i(k-1)}\right)\right] g_{i}\left(C_{i k}\right) .
$$

We refer to $L_{i}\left(z_{i}\right)$ as the total learning cost of language community $i$. Total learning cost is the integral over individual learning costs of all members of language community $i$. The total communication benefit $U_{i}(z)$ of language community $i$ is defined as follows:

$$
U_{i}(z)=\sum_{k=1}^{2}\left(z_{i k}-z_{i(k-1)}\right) U_{i}\left(C_{i k}, z_{-i}\right)
$$

for $i=1, \ldots, n$. Obviously, $U_{i}(z)$ is the integral over the communication benefits of all members of language community $i$. The group payoff $P_{i}(z)$ of language community $i$ is the difference between total communication benefit and total learning cost:

$$
P_{i}(z)=U_{i}(z)-L_{i}\left(z_{i}\right)
$$

for $i=1, \ldots, n$. The function $P_{i}$ which assigns $P_{i}(z)$ to every $z \in Z$ is the group payoff function of language community $i$. The function $P$ which assigns

$$
P_{z}=\left(P_{1}(z), \ldots, P_{n}(z)\right)
$$

to every $z \in Z$ is the vector payoff function. $P_{i}$ and $P$ are defined relative to a fixed combination $r=\left(r_{1}, \ldots, r_{n}\right)$ of cost compatible rankings. When we want to express this dependency, we shall write $P_{i}^{r}$ and $P^{r}$ instead of $P_{i}$ and $P$, respectively.

Aggregated games. For every combination $r=\left(r_{1}, \ldots, r_{n}\right)$ of cost compatible rankings, the associated aggregated game

$$
G^{r}=\left(Z, P^{r}\right)
$$

is an $n$-person game in normal form, where $Z_{1}, \ldots, Z_{n}$ are the strategy sets of the players $1, \ldots, s$, respectively, and where $P^{r}$ is the payoff function.

Best replies and equilibrium points. We use the notation $z_{i} z_{-i}$ in order to denote 
that border point system $z$ which contains $z_{i}$ and the components $z_{j}$ of $z_{-i}$ as components. A border point vector $\tilde{z}_{i} \in Z_{i}$ is a best reply to an $i$-incomplete border point system $z_{-i}$ in $G^{r}$ if we have:

$$
P_{i}^{r}\left(\tilde{z}_{i} z_{-i}\right)=\max _{z_{i} \in Z_{i}} P_{i}^{r}\left(z_{i} z_{-i}\right) .
$$

We say that $\tilde{z}_{i}$ is a best reply to a border point system $z$ in $G^{r}$ if in $G^{r}$ the border point vector $\tilde{z}_{i}$ is a best reply to the $i$-incomplete border point system $z_{-i}$ in $z$. We also say that the border point system $\tilde{z}$ is a best reply to $z$ in $G^{r}$ if every component of $\tilde{z}$ is a best reply to $z$ in $G^{r}$. An equilibrium point $z^{*}$ of $G^{r}$ is a border point system with the property that $z^{*}$ is a best reply to $z^{*}$ in $G^{r}$.

\section{Existence of equilibrium points}

In this section we shall prove the existence of equilibrium points for every game $\Gamma(m, n$, $a, b, f, g$ ) whose specification satisfies assumptions 1 to 5 . For this purpose we first show that an equilibrium point of the original game is associated with every equilibrium point of every aggregated game. We then turn our attention to the continuity and convexity properties of group payoffs, which later enable us to apply standard results from the literature to prove the existence of an equilibrium point for aggregated games.

Wherever we do not explicitly say anything to the contrary, statements refer to a fixed game $\Gamma(m, n, a, b, f, g)$ whose specification satisfies assumptions 1 to 5 and to a fixed combination of cost compatible rankings $r=\left(r_{1}, \ldots, r_{n}\right)$. The notation $G=(Z, P)$ is used for the associated game $G^{r}$. If we say that a border point vector or a border point system is a best reply, we mean a best reply in $G$.

Theorem 2 asserts the existence of equilibrium points for all aggregated games of $\Gamma(m$, $n, a, b, f, g)$ and therefore does not refer to a specific $r=\left(r_{1}, \ldots, r_{n}\right)$. Similarly, theorem 3 , the final existence theorem, refers to all games $\Gamma(m, n, a, b, f, g)$ satisfying assumptions 1 to 5 .

Lemma 4. Let $z_{i} \in Z_{i}$ be a best reply to $z_{-i} \in Z_{-i}$. Moreover, let $\varphi_{i}$ be a group strategy in the border equivalence class $\Lambda_{i}\left(z_{i}\right)$, and let $\varphi_{-i}$ be an $i$-incomplete strategy combination in the border equivalence class $\Lambda_{-i}\left(z_{-i}\right)$. Then $\varphi_{i}$ is an almost best reply to $\varphi_{-i}$. Moreover, for every $t \in\left[0, a_{i}\right]$ which does not coincide with one of the border points $z_{i k}$ in $z_{i}$, the strategy $\varphi_{i}(t)$ is a best reply of $i+t$ to $\varphi_{-i}$.

Proof. Assume that $\varphi_{i}$ is not an almost best reply to $\varphi_{-i}$. Then at least one of the open intervals of the form $\left(z_{i(k-1)}, z_{i k}\right)$ must contain infinitely many points $t$ where $\varphi_{i}(t)=C_{i k}$ is not a best reply of $i+t$ to $\varphi_{-i}$. Moreover, at least one strategy $D \in M_{i}$ must be a best reply 
of $i+t$ to $\varphi_{-i}$ for infinitely many of these values of $t$. Let $D$ be a strategy of this kind, and let $S$ be the set of all $t \in\left(z_{i(k-1)}, z_{i k}\right)$ such that $D$ is a best reply and $C_{i k}$ is not a best reply of $i$ $+t$ to $\varphi_{-i}$. For every $t \in S$ we have:

$$
U_{i}\left(D, \varphi_{-i}\right)-f_{i}(t) g_{i}(D)>U_{i}\left(C_{i k}, \varphi_{-i}\right)-f_{i}(t) g_{i}\left(C_{i k}\right)
$$

Essentially the same argument as has been applied to (17) and (18) in the proof of lemma 2 can be applied here, too. In this way it can be seen that $S$ is a subinterval of $\left(z_{i(k-1)}, z_{i k}\right)$. Moreover, since $S$ contains infinitely many points, $S$ has positive length. Let $\varphi_{i}^{\prime}$ be the following group strategy:

$$
\varphi_{i}^{\prime}(t)= \begin{cases}D & \text { for } t \in S \\ \varphi_{i}(t) & \text { for } t \notin S\end{cases}
$$

In view of (51) and the finite length of $S$, we obtain:

$$
\int_{0}^{a_{i}} H_{i+t}\left(\varphi_{i}^{\prime}, \varphi_{-i}\right) d t>P_{i}\left(z_{i} z_{-i}\right)
$$

According to lemma 3, the monotonicity class $\Psi_{i}\left(r_{i}\right)$ contains a best reply $\Psi_{i}$ to $\varphi_{-i}$. Let $z_{i}^{\prime}$ be the border point vector of a best reply $\Psi_{i} \in \Psi_{i}\left(r_{i}\right)$ to $\varphi_{-i}$. It follows by (53) that we have:

$$
P_{i}\left(z_{i}^{\prime} z_{-i}\right)>P_{i}\left(z_{i} z_{-i}\right)
$$

This is a contradiction to the assumption that $z_{i}$ is a best reply to $z_{-i}$. Therefore, $\varphi_{i}$ is an almost best reply to $\varphi_{-i}$.

We now prove the remaining assertion of the lemma. Suppose that one of the finitely many values of $t$, where $\varphi_{i}(t)$ is not a best reply to $\varphi_{-i}$, is a value $t_{0}$ which does not coincide with one of the border points. In some $\in$-neighborhood of $t_{0}$ all values of $t$ with the exception of $t_{0}$ have the property that $\varphi_{i}(t)=C_{i k}$ is a best reply of $i+t$ to $\varphi_{-i}$, where the strategy $C_{i k}$ is the same one for all these values of $t$. However, in view of lemma 2 the set of all $t$ such that $C_{i k}$ is a best reply of $i+t$ to $\varphi_{-i}$ is an interval. It must be that $t_{0}$ belongs to the interval. This is a contradiction. Therefore, the assertion holds.

Lemma 5. Let $z=\left(z_{1}, \ldots, z_{n}\right)$ be an equilibrium point of the aggregated game $G$. Then the border equivalence class $\Lambda(z)$ contains an equilibrium point.

Proof. Let $\varphi=\left(\varphi_{1}, \ldots, \varphi_{n}\right)$ be an element of $\Lambda(z)$. It follows by lemma 4 that $\varphi$ is an almost equilibrium point. Let $\Psi$ be related to $\varphi$ as in lemma 1 . It follows by lemma 1 that $\Psi$ is an equilibrium point. In view of lemma 4 , it is clear that $\Psi$ differs from $\varphi$ only at border points in the components of $z$. Consequently, $\Psi$ belongs to $\Lambda(z)$.

Lemma 6. For $i=1, \ldots, n$, the group payoff $P_{i}(z)$ is a continuous and bounded function of $z$, and for every fixed $z_{-i} \in Z_{-i}$ the group payoff $P_{i}\left(z_{i} z_{-i}\right)$ is a concave function of $z_{i}$. 
Proof. Since definite integrals of bounded functions are continuous in their upper limits, the functions $B_{i j}$ and $F_{i}$ are continuous. Therefore, the continuity of $P_{i}(z)$ as a function of $z$ is an immediate consequence of the definition of group payoffs. The boundedness of $P_{i}(z)$ follows by the compactness of $Z$.

In view of (46), for fixed $z_{-i} \in Z_{-i}$ the total community benefit $U_{i}(z)$ is a linear function of $z_{i}$. Therefore, it is sufficient to show that $L_{i}\left(z_{i}\right)$ is convex in $z_{i}$ in order to prove that $P_{i}\left(z_{i} z_{-i}\right)$ is concave in $z_{i}$. Equation (45) can be rewritten as follows:

$$
L_{i}\left(z_{i}\right)=\sum_{k=1}^{2} F_{i}\left(z_{i k}\right)\left[g_{i}\left(C_{i k}\right)-g_{i}\left(C_{i(k+1)}\right)\right]+a_{i} g_{i}\left(C_{i 2^{m-1}}\right) .
$$

Since $F_{i}\left(z_{i k}\right)$ is the integral over a monotonically increasing function, it is a strictly convex function of $z_{i k}$. In view of $g_{i}\left(C_{i k}\right) \leq g_{i}\left(C_{i(k+1)}\right)$, it follows by (55) that $L_{i}\left(z_{i}\right)$ is a convex function of $z_{i}$.

Theorem 2. For every combination $r=\left(r_{1}, \ldots, r_{n}\right)$ of cost compatible rankings, the associated aggregated game $G^{r}$ has at least one equilibrium point.

Proof. The strategy sets $Z_{i}$ are convex and compact. Lemma 6 has shown that $P_{i}^{r}(z)$ is continuous with respect to $z$ and concave with respect to player $i$ 's strategy $z_{i}$. Therefore, the conditions for the application of well known results on the existence of equilibrium points are satisfied (see, e.g., Friedman, 1977, p. 160, theorem 7.4). We can conclude that the assertion holds.

Theorem 3. Every game $\Gamma(m, n, a, b, f, g)$ whose specification satisfies assumptions 1 to 5 has at least one equilibrium point.

Proof. The assertion is an immediate consequence of lemma 5 and theorem 2.

\section{References}

Breton, Albert, \& Mieskowski, Peter (1975). The returns to investment in language: the economics of bilingualism. Toronto: University of Toronto, Institute for Policy Analysis.

Dressler, Wolfgang U. (1982). “Acceleration, retardation, and reversal in language decay?". In Language spread, ed. Robert L. Cooper (Bloomington, IN: Indiana University Press), pp. 321-336.

Friedman, James W. (1977). Oligopoly and the theory of games. Amsterdam: NorthHolland.

Grenier, Gilles (1985). "Bilinguisme, transferts linguistiques et revenus de travail au Québec: quelques éléments d'interaction. In Économie et langue, ed. François Vaillancourt (Québec: Éditeur officiel), pp. 243-287.

Hočevar, Toussaint (1975). "Equilibria in linguistic minority markets". Kyklos, 28, 337357.

Lang, Kevin (1986). “A language theory of discrimination”. The quarterly journal of 
economics, 100, 363-381.

Laponce, Jean (1984). Langue et territoire. Québec: Les presses de l'Université Laval.

Lieberson, Stanley (1982). "Forces affecting language spread: some basic propositions". In Language spread, ed. Robert L. Cooper (Bloomington, IN: Indiana University Press), pp. 37-62.

Sabourin, Conrad (1985). "La théorie des environnements linguistiques". In Économie et langue, ed. François Vaillancourt (Québec: Éditeur officiel), pp. 59-82.

Vaillancourt, François (1985). "Les écrits en économie de la langue: brève revue et introduction au recueil”. In Économie et langue, ed. François Vaillancourt (Québec: Éditeur officiel), pp. 11-25.

Vaillancourt, François (1985a). "Le choix de la langue de la consommation". In Économie et langue, ed. François Vaillancourt (Québec: Éditeur officiel), pp. 209-220.

Vaillancourt, François, \& Lacroix, Robert (1985). "Revenus et langue au Québec, 1970_ 1980: une revue des écrits". In Économie et langue, ed. François Vaillancourt (Québec: Éditeur officiel), pp. 221-242. 